\title{
Letter \\ Describing, Step by Step, the Shroud Body Image Formation
}

\author{
Giovanni Fazio ${ }^{1, *,+}$, Giuseppe Mandaglio ${ }^{2,3,+}$ and Antonio Anastasi ${ }^{1,+}$ \\ 1 Dipartimento di Scienze Matematiche ed Informatiche, Scienze Fisiche e Scienze della Terra, \\ Università di Messina, I-98166 Messina, Italy; antonioanastasi89@gmail.com \\ 2 Istituto Nazionale di Fisica Nucleare, Sezione di Catania, I-95123 Catania, Italy; gmandaglio@unime.it \\ 3 Dipartimento di Scienze Chimiche, Biologiche, Farmaceutiche e Ambientali, Università di Messina, \\ I-98166 Messina, Italy \\ * Correspondence: gfazio@unime.it \\ + These authors contributed equally to this work.
}

Received: 20 November 2018; Accepted: 20 December 2018; Published: 22 December 2018

\begin{abstract}
In the recent past, we introduced a natural mechanism (stochastic) in the literature to explain the image formation on the Linen of Turin by taking into account the yellowed linen fibrils distribution of the Shroud. In our opinion, a weak energy source like the thermal radiation produced by the enveloped corpse yielded on the linen a latent image, ruled by a stochastic process, and made by small bundles of yellowed fibrils. The image on the Shroud is unique and it appeared later than the blood image with its 2D and 3D characteristics. Moreover, a correlation between the density of yellowed fibrils and the cloth-body distance is present, again proving the action of a stochastic process. In this paper, we would like to discuss, step by step, the whole natural process that occurred in the formation of the Shroud body image. In every case, to confirm our hypothesis of Shroud body image formation, it is necessary to perform experimental investigations in the Forensic Medicine area.
\end{abstract}

Keywords: Thermal energy; Stochastic process; Yellowed fibrils; Shroud body image formation

\section{Introduction}

In Turin Cathedral, there is an old rectangular piece of unbleached linen $(441 \mathrm{~cm} \times 113 \mathrm{~cm})$ known as the "Shroud of Turin". This ancient cloth (with a history confirmed from the14th Century, when it was in the possession of Geoffrey de Charney in Lirey, France) bears the frontal and dorsal image of a man scourged and crucified. In fact, placed in appropriate areas of the body image, there are bloodstains. Moreover, the linen cloth shows burned and scorched areas, several stains called "water marks", and holes where a fire has burnt through the material.

Many people venerate this cloth as the burial one of Jesus of the Gospels. Others denounce it as a Medieval forgery. In 1452, the above cloth was ceded to duke Ludwig I of Savoy who, in 1502, placed it in the "Sainte Chapelle" of Chambery. Here, in 1532, a fire caused the above cited scorched and burned areas. In 1983, Humbert II of Savoy gave the Shroud to the Roman Catholic Church.

The STURP (The Shroud of Turin Research Project) team in its conclusions has written: " ... the Shroud image is that of a real human form of a scourged, crucified man. It is not the product of an artist". Besides, the image on the Shroud seems to be "latent" (Pellicori-Evans). In fact, the fibrils extracted from the bloodstains and serum, that were arranged as occurs for a cloth used to envelope a wounded human body, when clean of the proteins, appeared as a white color, not yellow. Therefore, the bloodstains were on the linen before the body image formation. Moreover, because of the serum permeability to the light, it is possible to observe a hint of the absence of the image below the yellowed linen (Miller-Pellicori). 
A few years ago, analyzing the uneven superficial distribution of the yellowed fibrils on the Shroud body image region, we thought about an interaction between the corpse that emits and the linen cloth that absorbs, deducing that [1]:

1. The probability of a fibril yellowing is a function of the energy;

2. The optical density of the fibrils is not a function of the energy.

In fact, in the areas of cloth-body contact, the number of yellowed fibrils per unit of surface (yellowed fibrils density or image intensity) is high. Additionally, the density of the yellowed fibrils linearly decreases as the distance between the cloth and the body increases. When the cloth-body distance is $\mathrm{R}_{0}=37 \mathrm{~mm}$ (valid only for the frontal image), the number of yellowed fibrils for unit area drops to zero. This means that in the Linen of Turin, there exists a correlation between the yellowed fibrils' density and the cloth-body distance [2]. The best function representing this correlation is a straight line. However, in scientific literature, a different correlation function with an exponential trend was proposed [3]. We do not agree with this last result because if it was true, the off-image area would also appear with yellowed fibrils, while we observe that this does not happen. The optical density of these fibrils is constant, in all the regions where the body image lies, with some variations due to the natural differences in the linen threads and/or in the manufacturing procedure [4].

We do not consider the dorsal image because the expected cloth-body distance is very small for the compression of the body on the cloth. Therefore, it is very difficult to measure and correlate the pairs' image intensity values and such distances for a fitting procedure. However, for the dorsal image, we can write: (1) the chemical modifications are the same as the ones present in the frontal image; (2) the thickness of the yellowed fibrils forming the images is the same in both the frontal and dorsal image; and (3) in the cloth-body contact areas, the intensity of the image is about the same in both images [2].

In this paper, we want to discuss the source triggering the process of formation, the above mechanism, and the presence of yellowed fibrils yielding the body image with its characteristics and uniqueness. In the synthesis, we want to highlight the various steps of the Shroud body image formation through a natural process.

\section{Thermal Energy Source}

We have always thought that the Shroud body image has been yielded by the action of a stochastic process evident when the available energy is very small and it is only not present if the absorbed energy is zero. In the case of our concern, we have considered a non-stationary heat transfer, naturally emitted by the enveloped corpse in the Linen of Turin.

Part of this energy is emitted per unit of body surface and time $\left(R=\varepsilon \cdot \sigma \cdot T^{4}\right)$ by the irradiation process. This contribution is small because the emissivity power of the human skin $\varepsilon$ is about 0.95 and the Stefan-Boltzmann constant $\sigma$ is very small $\left(5.67 \cdot 10^{-8} \cdot \mathrm{W} \cdot \mathrm{m}^{-2} \cdot \mathrm{K}^{-4}\right)$, as it is necessary to trigger a stochastic process.

The expression of the speed of energy transfer through the $S$ surface $(d Q / d t=-c \cdot S \cdot d T / d z)$, which represents the heat transfer by conduction, shows a temperature gradient that is very high because the thermal conductivity $\mathrm{c}$ of the linen is very low $\left(5 \cdot 10^{-4} \cdot \mathrm{cal} \cdot \mathrm{cm}^{-1} \cdot \mathrm{s}^{-1} \cdot \mathrm{K}^{-1}\right)$, in line with the thickness of the body image that is about two or three fibrils (20-30 microns [5]).

Finally, the expression of the heat transfer by convection (contributed by areas where cloth and body are not in contact) is known as the Newton law of cooling $d Q / d t=h_{c}$ mean $\cdot A \cdot\left(T_{\text {corpse }}-T_{\text {air }}\right)$, where $h_{c, \text { mean }}$ represents the mean convective coefficient of thermal exchange. This last parameter is very difficult to calculate because it is dependent on many parameters, such as viscosity, density, thermal conductivity, and specific heat (characteristics of the fluid and temperature dependent). It also depends on the geometry and velocity of the fluid (laminar motion or turbulent motion, by the knowledge of the Reynolds number). Consequently, we estimated, in a crude manner, the Nusselt number representing the ratio between the heat flux by convection and the one by conduction. 
We obtained values ranging between 5 and 30, in agreement with a strong presence of flux by convection with respect to the one by conduction. However, because the depth of the body image is independent from the cloth-body distance, we could deduce that in the areas without contact, the heat transfer by convection with a small amount of heat transfer by conduction yielded the same effects of only conduction in the contact zones.

\section{Mechanism of the Shroud Body Image Formation}

In this chapter, for a better comprehension of the mechanism of the Shroud body image formation, we propose a parallel between people exposed to a low radiation dose and linen fibrils of the Shroud. The effects of radiation on the exposed population are stochastic, with the following characteristics:

1. a threshold-dose does not exist;

2. the effects are only absent when the absorbed dose is equal to zero;

3. the effects have a probabilistic nature;

4. the frequency of appearance increases with the dose;

5. the effects appear years or decades later;

6. the seriousness of the above effects does not increase with the dose;

7. these effects are indistinguishable from the ones induced by other causes.

Moreover, the stochastic effects on the irradiated population show a correlation between the number of sick people $\mathrm{E}$ and the absorbed dose $\mathrm{D}$. This function is a straight-line and in the (E, D) plane, passes through the origin. The above affirmations are demonstrated by radiologic experiments and by the epidemiological evidence [6,7].

In our proposed parallel, the yellowed fibrils of the Linen of Turin are distributed with the same trend as that of the dead persons (years or decades after the radiation exposition) in an irradiated population with a low absorbed dose. This result has been obtained by the comparison between many hundreds of thousands of irradiated persons [6,7] and the approximately $3 \times 10^{7}$ fibrils (yellowed and with background color) in the image area. Indeed, in the Shroud fabric, we can hypothesize 2000-2200 fibrils $/ \mathrm{cm}^{2}$ [8-10]. So, by considering both the linen and the body image dimensions, we roughly obtain the above number.

In fact, in our parallel, the persons who are not sick correspond to the fibrils not yellowed. After radiation exposition, years or decades later, part of population dies from cancer, while some of the fibrils become yellow. In both cases, there are chemical changes. Consequently, in the presence of the stochastic process that we have described, it is natural to put persons and fibrils, sick persons and yellowed fibrils, and also ionizing radiation dose and absorbed thermal energy in parallel. This parallel helps, in our opinion, to understand the stochastic process behind the Shroud body formation.

As written in the introduction, the yellowed fibrils' distribution on the Shroud is the result of the action of a stochastic process. Indeed, the characteristics of the above distribution are in line with the (i) and (ii) items $[1,11,12]$. Moreover, to confirm this action, there is a correlation between the density of the above fibrils and the cloth-body distance. This function is represented by a linear regression. The result, on the Linen of Turin, appears with the fibrils grouped in small bundles near areas with background color. This complex distribution of yellowed fibrils, yielding the body image, can be observed (see Figure 1) in the micro-photographs taken in 1978 by Evans $[13,14]$.

It is known that a stochastic process produces visible effects after years or decades. Therefore, this could have been the case for the Shroud 'latent' body image [1,11]. The 2D (image in reversed color with left and right exchanged) and 3D (image with cloth-body distance information, codified in the light and dark areas distribution, allowing a reconstruction, in relief, with weak distortions) characteristics of the image on the Linen of Turin are well-explained by the process of our concern [11]. 


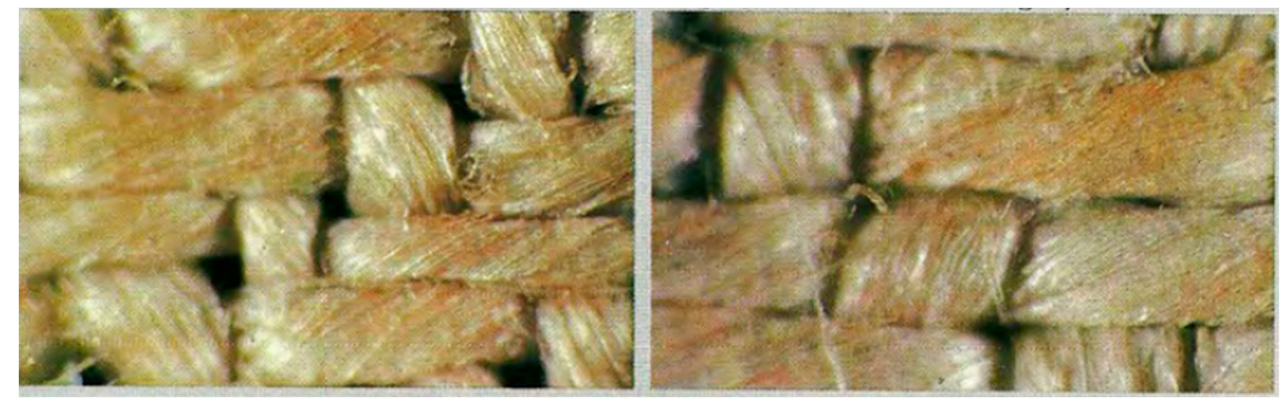

Figure 1. Microphotographs taken by Barrie M. Schwortz Collection, STERA Inc. (left) The dorsal heel area at $30 \times$ magnification; (right) tip of the nose at $36 \times$ magnification.

Besides, in the case of the enveloped corpse in the Linen of Turin, to obtain the result by the action of a stochastic process, it is necessary to remove the body from the Shroud before the emission of the first amines [15]. Indeed, with this hypothesis, the above emitted gases cannot have interactions with the fabric or, eventually, with the chemical substances that, in precedence, could have been distributed on it. In a recent article, some forensic experts [16] have shown how difficult it would be to get an image with details visible in the Shroud body image. Only if the corpse, from the beginning of the first amines emission, has been removed from the Linen of Turin, will we be able to justify the uniqueness of the Shroud body image.

The uneven superficial distribution of the yellowed fibrils on the Shroud yields an image with a density that is a function of the cloth-body distance. This distribution is the result of the energy transfer during the interaction between the emitting corpse and the absorbing linen. So, in the contact area, where the energy transfer between the corpse and the linen fabric is at the maximum, many fibrils (all in the same manner) increased their optical density. Differently, in the areas where the cloth-body distance is different from zero, the absorbed energy is smaller and the fibrils reaching the above optical density are less numerous.

\section{Conclusions}

The Shroud of Turin, due to its uniqueness and its connection with religion, has attracted the interest of large and different scientific and non-scientific communities. Some people believe that the Shroud is a Medieval forgery and have tried to demonstrate this hypothesis with experiments in the laboratory, whilst others believe that the Shroud is the product of a miraculous event. We propose, in the present paper and in our previous publications, a natural mechanism to explain the Shroud body image formation: the yellowed fibrils yielding the image show a probabilistic distribution that can be described with a mathematical expression: $\mathrm{P}(\mathrm{z})=\mathrm{P}_{\max } \cdot\left(1-\mathrm{z} / \mathrm{R}_{0}\right)$, where $\mathrm{P}(\mathrm{z})$ is the probability to yellow a fibril at the $z$ cloth-body distance, $P_{\max }$ is the maximum probability (measurable in the areas with $z=0)$, and $R_{0}\left(37 \mathrm{~mm}\right.$, only for the frontal image) is the $\mathrm{z}$ value that makes $P\left(R_{0}\right)=0$ [17].

The process that yielded the latent image on the Linen of Turin, made of small bundles of yellowed fibrils, is, in our opinion, natural: the weak body-linen cloth transmission of thermal energy occurred through all the three heat transfer mechanisms. Indeed, the conduction, convection, and irradiation have contributed, yielding an uneven superficial distribution of yellowed fibrils.

In every case, this process must be verified by experiments in the Forensic Medicine area by taking into account the long latency time of the image formation (years or decades).

Author Contributions: The authors contributed equally in all the various phases of the work.

Funding: This work has been made without external funding.

Acknowledgments: On this occasion, the authors wish to remember Raymond N. Rogers (1927-2005). A point of reference in our relationship with the Shroud of Turin. We are grateful to him for what we have learned from his writings.

Conflicts of Interest: The authors declare no conflict of interest. 


\section{References}

1. Fazio, G.; Mandaglio, G. Stochastic distribution of the fibrils that yielded the Shroud of Turin body image. Radiat. Effects Defects Solids 2011, 166, 476-479. [CrossRef]

2. Jackson, J.P.; Jumper, E.J.; Ercoline, W.R. Correlation of image intensity on the Turin Shroud with the 3D structure of a human body shape. Appl. Opt. 1984, 23, 2244-2270. [CrossRef] [PubMed]

3. Fanti, G. Can a corona discharge explain the body image of the Turin Shroud? J. Imaging Sci. Technol. 2010, 54, 20508-1-20508-11. [CrossRef]

4. Rogers, R.N. A Chemist's Perspective on the Shroud of Turin; Joan Rogers \& Barrie M. Schwortz: Florissant, CO, USA, 2008.

5. Schwalbe, L.A.; Rogers, R.N. Physics and chemistry of the Shroud of Turin. A Summary of the1978 Investigation. Anal. Chim. Acta 1982, 135, 3-49. [CrossRef]

6. Moe, H.J.; Lusak, S.R.; Schumaker, M.C. Radiation Safety Technician Training Course Argonne National Laboratory; Industrial Hygiene and Safety Division: Chicago, IL, USA, 1971.

7. Obodovskiy, I. Fundamentals of Radiation and Chemical Safety; Elsevier Inc.: Amsterdam, The Netherlands, 2015.

8. Tyrer, J. Looking of the Turin Shroud as a textile. Text. Horiz. Available online: http:/ /www.sindone.info/ TYRER1.PDF (accessed on 22 December 2018).

9. Little, K. The formation of the Shroud's body image. BSTS Nerwsl. 1997, 46, 22-26.

10. Di Lazzaro, P. Un Approccio Scientifico alla Formazione Dell'immagine Della Sindone. 2011, pp. 1-9. Available online: https:/ / www.uccronline.it/2011/10/30/un-approccio-scientifico-alla-formazione-dell\% E2\%80\%99immagine-della-sindone-iio-parte/ (accessed on 30 October 2011).

11. Fazio, G.; Mandaglio, G. Can a latent image explain the characteristics of the Shroud body image? Radiat. Effects Defects Solids 2012, 167, 220-223. [CrossRef]

12. Fazio, G.; De Leo, V.; Curciarello, F.; Mandaglio, G. Comparison among the Shroud body image formation mechanisms by the linen fibrils distributions. J. Text. Inst. 2015, 106, 896-899. [CrossRef]

13. Pellicori, S.F.; Evans, M.S. The Shroud of Turin through the microscope. Archaeology 1981, 34, $34-43$.

14. Jumper, E.J.; Adler, A.D.; Jackson, J.P.; Pellicori, S.F.; Heller, J.H.; Druzik, J.R. A comprehensive examination of the various stains and images on the Shroud of Turin. ACS Adv. Chem. Archaeol. Chem. III 1984, 20, 447-476.

15. Vass, A.A.; Barshick, A.; Sega, G.; Caton, J.; Skeen, J.T.; Love, J.C.; Synstelien, J.A. Decomposition chemistry of human remains: A new methodology for determining the postmortem interval. J. Forensic Sci. 2002, 47, 542-553. [PubMed]

16. Tattoli, L.; Tsokos, M.; Buschmann, C. Could the Shroud of Turin be an effect of post-mortem changes? Forensic Sci. Med. Pathol. 2014, 10, 469-471. [CrossRef] [PubMed]

17. Fazio, G.; Mandaglio, G.; Anastasi, A. Revisiting a pure stochastic mechanism to explain the body image formation on the Linen of Turin. J. Text. Inst. 2017, 108, 1552-1555. [CrossRef]

(C) 2018 by the authors. Licensee MDPI, Basel, Switzerland. This article is an open access article distributed under the terms and conditions of the Creative Commons Attribution (CC BY) license (http:// creativecommons.org/licenses/by/4.0/). 